\title{
THORACOSCOPY IN ACQUIRED IMMUNODEFICIENCY SYNDROME
}

David R. Flum, MD

Scott D. Steinberg, MD

Thomas R. Bernik, MD

Enrique Bonfils-Roberts, MD

Marshall D. Kramer, MD

Peter X. Adams, MD

Marc K. Wallack, MD
Objective: The role of thoracic surgery in patients with acquired immunodeficiency syndrome (AIDS) continues to evolve. This review seeks to evaluate the outcome, morbidity, and mortality associated with videoassisted thoracoscopic surgery for empyema and pneumothorax in patients with AIDS. Methods: A retrospective review was conducted of patients with AIDS in whom video-assisted thoracoscopic surgery was performed for empyema (group 1) or intractable pneumothorax (group 2). Results: Twenty patients with AIDS (95\% male, mean age 37.4 years, mean CD4 count 76 cells $/ \mathrm{ml}^{3}$ ) underwent thoracoscopy. Surgery was performed for empyema (group 1) in $11(55 \%)$ and intractable pneumothorax (group 2) in nine (45\%). Three patients (15\%) died within 30 days of the operation. At mean follow-up (29 months), overall survival was $55 \%$. For those who survived the hospitalization and died within the follow-up period $(35.3 \%)$, mean survival time was 8.2 months (range 1 month to 27 months). In group 1, surgical procedures were performed after 8 days of chest tube drainage and included pleural debridement and mechanical pleurodesis $(n=11)$ along with lung biopsy $(n=6)$. Survivals at 30 days and 29 months' follow-up were $90.9 \%$ and $45.4 \%$, respectively. In group 2 , significantly depressed CD4 counts (average 33.2 cells $/ \mathrm{ml}^{3}$ ) were noted along with a more prolonged preoperative hospitalization (18.5 days) with 14.2 days spent with a chest tube before the operation. In this group, operative procedures included mechanical pleurodesis and talc poudrage $(n=9)$, bleb resection $(n=7)$, and lung biopsy $(n=1)$. Two deaths $(22 \%)$ occurred within 30 days of the operation and survival at 29 month' follow-up was $66 \%$. Conclusion: Video-assisted thoracoscopic surgery performed in patients with AIDS for the treatment of empyema and intractable pneumothorax is effective, can be performed with little operative morbidity and mortality, and is associated with acceptable long-term survival. Video-assisted thoracoscopic surgery is best performed soon after the diagnosis of intractable pneumothorax or empyema has been established. (J Thorac Cardiovasc Surg 1997;114:361-6)
As the frequency of acquired immunodeficiency syndrome (AIDS) has increased, so too has the role of surgery in the spectrum of therapy. Although thoracic surgeons have been involved in the treatment of pulmonary complications of AIDS, the timing and utility of thoracic surgery in this population continues to develop. ${ }^{1,2}$ Pulmonary manifesta-

From St. Vincent's Hospital and Medical Center, New York Medical College, New York, N.Y.

Received for publication Jan. 10, 1997; revisions requested April 7, 1997; revisions received May 5, 1997; accepted for publication May 6, 1997.

Address for reprints: David R. Flum, MD, 130 West 12th St., 11G, New York, NY 10011.

Copyright (C) 1997 by Mosby-Year Book, Inc.

$0022-5223 / 97 \$ 5.00+0 \quad \mathbf{1 2 / 1 / 8 3 0 6 4}$ tions are common among patients with AIDS. Pneumocystis carinii pneumonia (PCP) is so closely associated with AIDS that its presence is diagnostic of the disease. PCP and other infectious agents have been linked to cavitary lesions in the lung parenchyma, tissue necrosis, and pneumothorax. Empyema is a periodic complication of pneumonia in this population, necessitating definitive drainage with tube thoracostomy. Incompletely drained empyema requires surgery for relief of symptoms and resolution of infection. Simple pneumothorax in patients with AIDS is most often treated by tube thoracostomy with success. However, intractable pneumothoraces refractory to multiple trials of closed suction have been identified. ${ }^{3,4}$ These refractory pneumothoraces periodically require thoracic surgi- 
cal intervention to facilitate the removal of chest tubes and discharge from the hospital.

Video-assisted thoracoscopic surgery (VATS) has gained in popularity as an alternative to thoracotomy in the treatment of pulmonary diseases. VATS has been used for early empyema, bleb resection, pleurodesis, pleural debridement for removal of empyema cavity debris, and talc poudrage. We have reviewed our experience with VATS in patients with AIDS to determine its role in the spectrum of therapy for the complications of AIDS.

\section{Methods}

A retrospective review was conducted of patients with AIDS who underwent VATS between September 1991 and April 1995. All inpatient records were reviewed and data regarding prehospital health status, demographics, comorbid factors, operative events, complications, and postoperative course were evaluated. Telephone contacts with all patients were conducted at various postoperative intervals.

Patients were included for consideration if they had AIDS-defining illnesses or an absolute T-cell count of less than $200 \mathrm{cells} / \mathrm{ml}^{3}$. Group 1 included patients undergoing surgery for empyema. This diagnosis was based on computed tomography of the chest demonstrating an incompletely drained fluid collection from which bacterial species were isolated. Group 2 included patients with intractable pneumothorax. This was defined as pneumothorax without complete resolution after more than two trials of 3 to 5 days of closed suction drainage. Intractable pneumothorax also described persistent air leak on chest tube suction for greater than 7 days.

Thoracoscopic procedures were performed with the aid of general anesthesia in all patients, and a doublelumen endotracheal tube was used for single lung ventilation during the procedure. VATS was performed through three $10 \mathrm{~mm}$ ports (Bluntport, United States Surgical Corporation, Norwalk, Conn.) in a standard fashion. Pleural debridement is described as removal of pleura-based exudative and fibrinous material along with division of pleural adhesions and irrigation of the empyema cavity. Mechanical pleurodesis refers to the abrasion of the parietal pleura. Talc poudrage describes the instillation of sterilized talc powder into the thoracic cavity. Parenchymal bullae were resected with Endo-GIA stapling equipment (United States Surgical Corporation).

All data were evaluated with a Microsoft Excel (version 4) statistical analysis package (Microsoft Corporation, Redmond, Wash.). Descriptive statistics and $t$ testing were used for group evaluation and to examine intergroup relationships. A $p$ value of $<0.05$ was considered statistically significant. Data are presented as means unless otherwise specified.

\section{Results}

Twenty patients with AIDS who were undergoing VATS at St. Vincent's Hospital and Medical Center of New York were studied. Ninety-five percent of the patients were men (mean age 37.4 years). The source of human immunodeficiency virus (HIV) infection was identified as sexual contact by $65 \%$ of the patients and intravenous drug use by $25 \%$. No source was provided in $10 \%$. The average CD4 count was $76 \mathrm{cells} / \mathrm{ml}^{3}$. Previous medical history was significant for PCP (55\%), Kaposi's sarcoma (30\%), Mycobacterium intracellularae (10\%), cytomegalovirus (25\%), Cryptosporidium (5\%), and toxoplasmosis $(5 \%)$. Eleven patients (55\%) had unilateral empyema (group 1). This diagnosis was based on computed tomography of the chest demonstrating an incompletely drained fluid collection from which bacterial species were isolated. In nine patients (45\%) intractable pneumothorax (group 2) was diagnosed. Intractable pneumothorax was defined as a recurrent pneumothorax after at least one trial of closed suction and waterseal drainage for 3 to 5 days for a spontaneous, primary pneumothorax.

VATS was performed in all patients without event. No operative complications occurred and no conversions to thoracotomy were necessary. The operation was considered successful in all patients. The average length of hospitalization was 38 days and the time from admission until the operation was performed was 17.6 days. The average length of time with a chest tube was 9.2 days before the operation. Thirty-five percent required intensive care unit stays. Three patients $(15 \%)$ died within 30 days of the operation. No wound healing complications were noted. At a mean follow-up of 29 months, overall survival was $55 \%$. For those who survived the hospitalization and died within the follow-up period (35.3\%), mean survival time was 8.2 months (range 1 to 27 months).

Empyema. Demographic characteristics were similar when comparisons were made between patients who had pneumothorax or empyema. Among patients undergoing surgery for empyema (group 1), the average CD4 count was $128 \mathrm{cells} / \mathrm{ml}^{3}$. The average length of time with a chest tube before the operation was 8 days and after the operation, 3.4 days. Surgical procedures included pleural debridement and mechanical pleurodesis $(n=11)$ and lung biopsy $(n=6)$. Pathologic evaluation of resected material revealed mixed bacterial species $(n=4)$, PCP $(n=2)$, Mycobacterium tuberculosis $(n=2)$, and no organisms $(n=3)$.

Three patients were admitted to the intensive care unit for postoperative evaluation. One patient required continued mechanical ventilation and died 
on postoperative day 23. Ten of $11(90.9 \%)$ patients were discharged to their homes. Four patients died after discharge from the hospital (mean 243 days). Overall survival at 29 months' follow-up was $45.4 \%$. No readmissions for thoracic surgical disease were necessary.

Pneumothorax. Patients who had surgery for persistent pneumothorax (group 2) had significantly depressed CD4 counts (average 33.2 cells $/ \mathrm{ml}^{3}$ ). A prolonged preoperative hospital admission was noted in this group (18.5 days), with a mean of 14.2 days spent with a chest tube before the operation. Seven patients had at least two trials of waterseal drainage before the operation. Three of nine patients $(33 \%)$ had the chest tube removed and replaced for recurrence of pneumothorax before the operation.

VATS was used to perform mechanical or talc pleurodesis, or both $(n=9)$, bleb resection $(n=7)$, and lung biopsy $(n=1)$. On average, two chest tubes were placed at the completion of the operation, and the last of these tubes remained in place for a mean of 4.1 days after the operation. Pathologic conditions included PCP $(n=7)$, cytomegalovirus $(n=$ $1)$, and Kaposi's sarcoma $(n=1)$.

Four patients were admitted to the intensive care unit after the operation ( $44 \%$ ) for respiratory insufficiency resulting in two operative deaths $(22 \%$, 30-day mortality). Two patients died within 12 months of hospital discharge with a calculated survival of $66 \%$ at 29 months' follow-up. No recurrent pneumothoraces were identified at follow-up.

\section{Discussion}

The role of the surgeon in dealing with the complications of HIV/AIDS continues to evolve. With growing experience the surgical community has identified many pathologic entities that are either reversible or ameliorated by surgery. One of the earliest reports of thoracic surgery in this group of patients evaluated thoracic surgical procedures (tracheostomy, repair of air leaks, mediastinoscopy, lobectomy, lung biopsy, and esophagogastrectomy) performed during 49 operations. ${ }^{1}$ This early evaluation recognized that certain thoracic procedures represented futile care whereas others provided effective symptomatic relief with limited morbidity.

In patients with AIDS the complications of pneumonia periodically require surgical intervention. These complications include complex pleural effusion, empyema, and pneumothorax. The operations typically are performed on an emergency basis and have not lent themselves to critical review. Thoracotomy for pneumothorax or empyema has been advocated by some, ${ }^{1}$ whereas others have suggested that minimally invasive surgery for patients with AIDS may be more suitable. ${ }^{2}$ In either case sparse data exist to support the role of surgery and no large surgical series has demonstrated the proper indications, predicted outcomes, and risk/benefit ratio of thoracic surgery in the patient with AIDS.

Coker and associates ${ }^{3}$ identified 87 patients with AIDS-related PCP, among whom 16 pneumothoraces were identified. Pneumothorax was linked to aerosolized pentamidine (for early treatment of PCP) and PCP alone in $58 \%$ and $83 \%$ of cases, respectively. In another series ${ }^{4}$ aerosolized pentamidine was associated with spontaneous pneumothorax in patients with AIDS. Pneumothorax was identified in 12 of 327 patients undergoing this therapy. Of these, $75 \%$ had active $\mathrm{PCP}, 83 \%$ required chest tube insertion, and $50 \%$ died. A retrospective review $^{5}$ found that the incidence of pneumothorax in patients with PCP was 8 of $89(9 \%)$ compared to $0 \%$ in patients without PCP. Prospective analysis ${ }^{6}$ of patients with AIDS determined the rate of pneumothorax to be 20 of 1030 patients (2\%). Of these, 19 had compelling evidence of PCP infection. Aerosolized pentamidine demonstrated a $17.6 \%$ increase in relative risk and PCP a $14.5 \%$ increased risk. The mortality rate associated with pneumothorax was $10 \%$. Gerein and coworkers ${ }^{7}$ described 22 patients with pneumothorax, of whom 17 died within the time frame of the study (average 147 days from initial pneumothorax therapy). In a similar report, 23 of 40 patients with pneumothorax died within 8 weeks of pneumothorax. ${ }^{8}$ An important subset of patients described by Byrnes, Brevig, and $\mathrm{Yeoh}^{9}$ is the group in whom pneumothorax developed while the patients were being supported by a ventilator; their mortality rate was $92 \%$.

The pathophysiology of PCP-related pneumothorax has been suggested from analysis of wedge resections of autopsy specimens. ${ }^{10}$ Tissue invasion with PCP and regional necrosis with cavitation was found in seven of eight patients with pneumothorax. Others $^{8}$ have found PCP-infected lung specimens that demonstrated an interstitial inflammatory process with subpleural necrosis and bullous change. Spontaneous rupture of such necrotic lung tissue is the suggested pathophysiology of pneumothorax. Tissue necrosis is likely related to poor tissue healing and refractory pneumothorax or air leak after conservative management. 
Despite the poor survival noted in early studies, some have reported reasonable improvements in outcomes after operations for pneumothorax. Gerein and associates ${ }^{7}$ described the results of eight of 22 patients with pneumothorax who underwent surgical procedures for resolution of pneumothorax. Open pleurectomy was performed in six with no recurrence noted. Although little follow-up was provided, a treatment algorithm was offered that emphasizes surgical pleurectomy for unsuccessful tube thoracostomy use or bilateral disease. This radical surgical approach has been reinforced by a case report ${ }^{11}$ that advocated median sternotomy for bilateral pneumothorax. In the largest series of surgical intervention for AIDS-related thoracic disease, Horowitz and Olive ${ }^{12}$ reported on seven patients undergoing eight open procedures for AIDS-associated pneumothorax. In this series, posterolateral thoracotomy was performed in five patients and sternotomy in two. No operative deaths were noted and mean time from operation until discharge was 13 days. Three patients died between 4 and 8 months after the operation.

The surgical community has increasingly applied minimally invasive technology to common thoracic procedures. Daniel, Tribble, and Rodgers ${ }^{13}$ described the use of VATS and talc poudrage in 40 patients. None of these patients had HIV infection or AIDS. A 95\% success rate was noted in 20 patients with spontaneous pneumothorax. Of 20 patients with intractable pleural effusions, $90 \%$ had successful outcomes. Minimally invasive surgery has particular advantages in those in whom the risk of thoracotomy is high. Despite the growing acceptance of this technique in coordination with bleb resection and pleurodesis, ${ }^{14}$ only scattered reports have described VATS in HIV/AIDS-related pneumothorax. Tunon-de-Lara and coworkers ${ }^{15}$ describe a single case of talc pleurodesis after pleuroscopic examination in a patient with AIDS. This patient died within 2 months of the operation. The only additional report ${ }^{16}$ detailed the thoracoscopic instillation of talc in a single patient with pneumothorax. No follow-up was provided.

The three phases of empyema development (exudative, fibrinopurulent, and organizing) have been well described ${ }^{17}$ and in large part determine the feasibility of surgical techniques for this disease. The early exudative phase of empyema is adequately treated with tube thoracostomy drainage. The fibrinopurulent stage of empyema most often requires surgical intervention to allow for resolution of infection, clearance of exudative and fibrinous debris, and complete reexpansion of the lung. In the organized phase of empyema development, a thick pleural "cortex" may trap the lung and most often requires a thoracotomy for removal. Although several authors ${ }^{18-20}$ have evaluated the role of thoracoscopy for the treatment of empyema, the role of VATS for patients with AIDS has not heretofore been described.

This report details the largest series, to date, of thoracoscopic surgery in patients with AIDS. Our institutional experience has generated an aggressive treatment algorithm (Fig. 1) for the management of this patient population. Patients with intractable pneumothorax (pneumothorax without resolution after at least one trial [ 3 to 5 days] of closed suction drainage) undergo thoracoscopic pleurodesis, resection of blebs when appropriate, and talc poudrage. Similarly, incompletely drained empyema is treated with surgery as early as that diagnosis is established and the patient's condition can be stabilized. For empyema, this time frame is usually 7 to 10 days after identification and once incomplete drainage from the chest tube has been recognized. Computed tomography of the chest is most often performed to assess the degree, location, and chronicity of the empyema cavity. For VATS to be performed adequately in patients with empyema, it is imperative that the operation be done before the development of a thick pleural "cortex" or significant lung trapping. The use of VATS for pleural debridement adequately allows for complete resolution of infection. ${ }^{18-20}$ Pleural debridement refers to the removal of fibrinous exudate and adhesions that form in the empyema cavity along with the resection of pleural "membranes" that might prevent complete reinflation of the lung. ${ }^{20}$ The ability to perform adequate decortication of a more organized empyema is limited by the thoracoscopic approach and associated with a higher likelihood of conversion to thoracotomy. ${ }^{18-20}$

Our results indicate that VATS can be applied with successful outcomes for both intractable pneumothorax and empyema. VATS is well tolerated by this patient population (30-day survival for group 1 was $91 \%$ and for group 2, 78\%). A better than expected rate of survival was noted at 2 years' follow-up (group 1, 45\%; group 2,66\%), especially when considering the extreme level of immunosuppression noted in this review. This is more significant when considering that most of these patients would be unable to leave the hospital (because of 


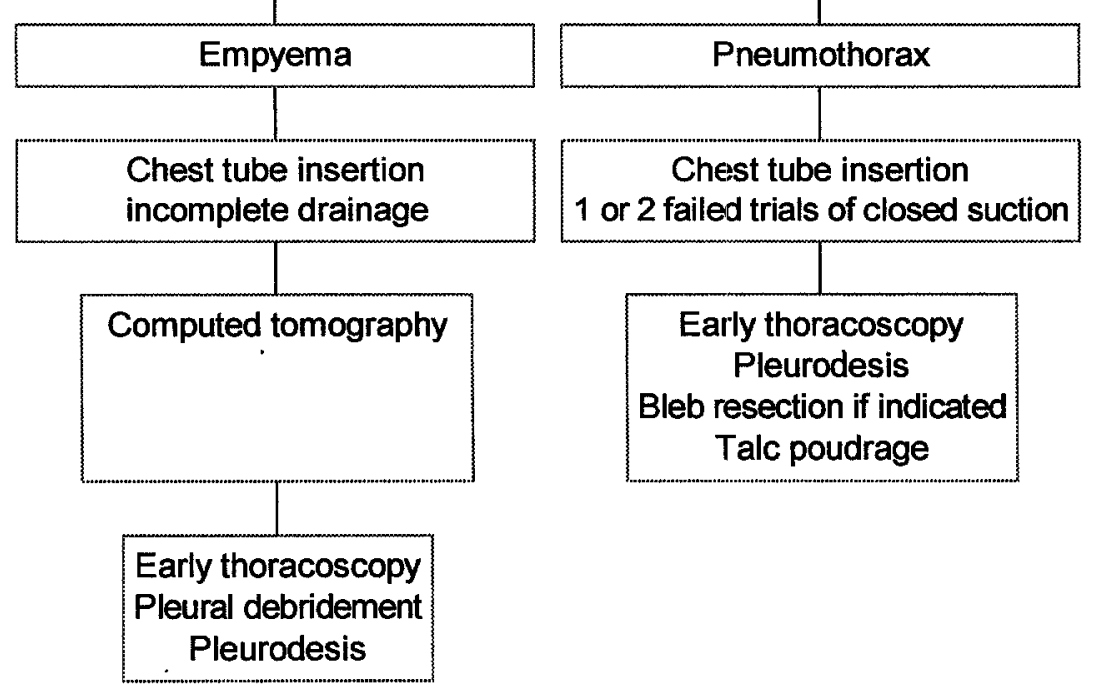

Fig. 1. Algorithm for the use of thoracoscopy in the treatment of patients with AIDS.

ongoing infection or chest tubes necessitating suction) unless surgical intervention was applied. Chest tubes are removed within 3 to 4 days after the operation. This is contrasted to the prolonged periods of time with chest tubes before the operation (group 1, 8 days; group 2, 14 days).

We have identified few predictors of survival after thoracoscopic surgery; however, patients in whom VATS is performed for empyema tended to require fewer intensive care unit admissions and were discharged from the hospital more rapidly if the operation was performed less than 7 days after chest tube insertion. As well, patients in whom VATS was performed for pneumothorax tended to require fewer days in the hospital after the operation and to have improved outcomes when the operation was performed after the first trial of closed suction drainage rather than after repeated trials. These relationships did not achieve statistical significance. Lower CD4 counts appeared to be linked to worsened postoperative survival but were not independent, predictive variables when evaluating for surgical risk. Indeed, we were surprised by the profound level of immunosuppression identified in patients undergoing operations for pneumothorax (group 2) and the low level of postoperative morbidity and mortality in this group.

Several nonsurgical alternatives exist for the treatment of patients with intractable pneumothorax and empyema. ${ }^{21}$ To avoid operative interventions, some have suggested leaving chest tubes in place for more prolonged periods, attaching one- way (Heimlich) valves for those with persistent air leaks, or applying bedside chemical pleurodesis. For empyema, enzymatic debridement has been offered as an alternative therapy. In general, the least morbid and most effective therapeutic intervention should be considered in all patients with pulmonary disease. In patients with AIDS this is complicated by associated comorbid disease and the higher than expected 30-day mortality identified in this study. However, it has been our experience that for both intractable pneumothorax and pleural effusion/empyema, early surgical intervention using the thoracoscope is the least morbid and most effective available therapy. Although this study did not compare operative and nonoperative therapy, such an evaluation would be helpful. It is our impression that the mortality involved in thoracoscopy has less to do with the procedure than with the late stage of presentation of these complications of AIDS. A further challenge is to identify prognostic indicators of poor outcome to allow better patient selection for surgical therapy. For example, patients who required mechanical ventilation for associated respiratory insufficiency were not offered VATS intervention.

Although no direct comparisons between thoracoscopic surgery and alternative therapy have been reported, we have observed that surgery is the more definitive and consistently successful therapy. Trachiotis and associates ${ }^{21}$ have reported good outcomes using an algorithm that emphasizes conservative management (Heimlich valves) over surgical 
therapy for intractable pneumothorax in patients with AIDS. Although these patients may leave the hospital, there are compelling reasons ${ }^{22}$ to avoid Heimlich valves. Leaving chest tubes with open drainage in patients with AIDS may pose a health risk to the patient and the community. ${ }^{22}$ The risk of empyema with persistent chest tube and Heimlich valve may be higher than expected. Most important, unlike Heimlich valves, thoracoscopy offers a chance for these chronically ill patients to leave the hospital and live their remaining days without chest tubes. We do not routinely use talc slurry for pneumothorax because experience has shown us that PCPrelated pneumothorax does not consistently respond to this therapy. Last, enzymatic debridement of loculations in early empyema may have a role in the spectrum of therapy. However, our experience suggests that, as in patients without HIV infection, early and complete reinflation of the lung and obliteration of the infected pleural space remains the best available therapy. For these reasons the suggested algorithm emphasizes early thoracoscopic management in patients with intractable pneumothorax or empyema.

Thoracoscopy for the complications of AIDSrelated pulmonary manifestations is well tolerated, adequately resolves the pathologic condition, and is associated with favorable outcomes. Only by evaluating the utility of surgical interventions can the medical community adequately serve its patients. Thoracoscopy for intractable pneumothorax and empyema has a clear role in the spectrum of AIDS.

\section{REFERENCES}

1. Miller JI. The thoracic surgical spectrum of acquired immune deficiency syndrome. J Thorac Cardiovasc Surg 1986;92:97780.

2. Feins RH. The role of thoracoscopy in the AIDS/immunocompromised patient. Ann Thorac Surg 1993;56:649-50.

3. Coker RJ, Moss F, McCarty M, et al. Pneumothorax in patients with AIDS. Respir Med 1993;87:43-7.

4. Newsome GS, Ward DJ, Pierce PF. Spontaneous pneumothorax in patients with acquired immunodeficiency syndrome treated with prophylactic aerosolized pentamadine. Arch Intern Med 1990;150:2167-8.

5. McClellan MD, Miller SB, Parsons PE, Cohn DL. Pneumo- thorax with Pneumocystis carinii pneumonia in AIDS. Chest 1991;100:1224-8.

6. Sepkowitz KA, Telzak EE, Gold JWM, et al. Pneumothorax in AIDS. Ann Intern Med 1991;114:445-59.

7. Gerein AN, Brumwell ML, Lawson LM, Chan NH, Montaner JSG. Surgical management of pnéumothorax in patients with acquired immunodeficiency syndrome. Arch Surg 1991;126:1272-7.

8. Beers MF, Sohn M, Swartz M. Recurrent pneumothorax in AIDS patients with pneumocystis pneumonia. Chest 1990;98: 266-70.

9. Byrnes TA, Brevig JK, Yeoh CB. Pneumothorax in patients with acquired immunodeficiency syndrome. $\mathbf{J}$ Thorac Cardiovasc Surg 1989;98:546-50.

10. Murry CE, Schmidt RA. Tissue invasion by Pneumocystis carinit: a possible cause of cavitary pneumonia and pneumothorax. Hum Pathol 1992;23:1380-7.

11. Fleisher AG, McElvaney G, Lawson L, Gerein AN, Grant D, Tyers GFO. Surgical management of spontaneous pneumothorax in patients with acquired immunodeficiency syndrome. Ann Thorac Surg 1988;45:21-3.

12. Horowitz MD, Olive H. Pneumothorax in AIDS: operative management. Am Surg 1993;59:200-4.

13. Daniel TM, Tribble CG, Rodgers BM. Thoracoscopy and talc poudrage for pneumothoraces and effusions. Ann Thorac Surg 1990;0:186-9.

14. Wakabayashi A. Thoracoscopic ablation of blebs in the treatment of recurrent or persistent spontaneous pneumothorax. Ann Thorac Surg 1989;48:651-3.

15. Tunon-de-Lara JM, Constans J, Vincent MP, Receveur MC, Conri C, Taytard A. Spontaneous pneumothorax associated with Pneumocystis carinii. Chest 1992;101:1177-8.

16. Kimmel RD, Karp MP, Cascone JJ, Zinns JS. Talc pleurodesis during videothoracoscopy for Pneumocystis carinii pneumonia-related pneumothorax. Chest 1994;105:314-5.

17. American Thoracic Society: Medical Section of the National Tuberculosis Association. Management of non-tuberculous empyema: a statement of the subcommittee on surgery. Am Rev Respir Dis 1962;85:935-6.

18. Landreneau RJ, Keenan RJ, Hazelrigg SR, Mack MJ, Naunheim KS. Thoracoscopy for empyema and hemothorax. Chest 1995;109:18-24.

19. Ferguson MK. Thoracoscopy for empyema, bronchopleural fistula and chylothorax. Ann Thorac Surg 1993;56:644-5.

20. Ridley PD, Braimbridge MV. Thoracoscopic debridement and pleural irrigation in the management of empyema thoracis. Ann Thorac Surg 1991;51:461-4.

21. Trachiotis GD, Vricella LA, Alyono D, Aaron BL, Hix WR. Management of AIDS-related pneumothorax. Ann Thorac Surg 1996;62:1608-13.

22. Thomas AN. Management of AIDS-related pneumothorax. Invited commentary. Ann Thorac Surg 1996;62:1613. 